\title{
Exploring Influencing Factors of Passenger Satisfaction toward Bus Transit in Small-Medium City in China
}

\author{
Xiaowei Li, ${ }^{1}$ Jingkun Fan, ${ }^{1}$ Yao Wu $\left(D,{ }^{2}\right.$ Jun Chen, ${ }^{1}$ and Xuefeng Deng ${ }^{1}$ \\ ${ }^{1}$ School of Civil Engineering, Xi'an University of Architecture \& Technology, Xi'an 710055, China \\ ${ }^{2}$ School of Transportation, Southeast University, Nanjing 210096, China \\ Correspondence should be addressed to Yao Wu; wuyaomaster@126.com
}

Received 24 September 2020; Revised 1 November 2020; Accepted 11 November 2020; Published 23 November 2020

Academic Editor: Maria Alessandra Ragusa

Copyright (c) 2020 Xiaowei Li et al. This is an open access article distributed under the Creative Commons Attribution License, which permits unrestricted use, distribution, and reproduction in any medium, provided the original work is properly cited.

This paper aims to explore the factors influencing passengers' satisfaction toward bus transit and develop the improvement strategy for the small-medium city. Data including individual attributes, travel activity attributes, and perceived service attributes were collected in the city of Weinan, China. The ordered logit (OL) model and ordered Probit (OP) model were employed to explore the significant factors associated with passengers' satisfaction toward bus transit. The odds ratio (OR) was applied to quantitatively measure the effects of the significant factors. Improving strategies of bus transit service were proposed based on the model results using the three-factor theory. Results show that the OL model outperforms the OP model. The age, daily average waiting time, perceived waiting time, transferring convenience, the attitude of the driver, intelligent travel information service, hygienic environment inside the bus, ticket price, bus route setting, and bus stop setting significantly affect the passengers' satisfaction. Among them, the ticket price, perceived waiting time, bus stop setting, intelligent travel information service, transferring convenience, and bus route setting were identified as exciting factors. It is recommended that optimization of bus route and bus stop setting, building bus dedicated lanes, optimizing dynamic charging system, and providing intelligent travel information service could be effective strategies to improve passengers' satisfaction toward bus transit in Chinese smallmedium cities.

\section{Introduction}

With the expansion of the urban scale and the rapid increase in traffic demand, traffic congestion has been a serious issue in China. In 2012, the Chinese government had issued urban public transport priority strategies for alleviating traffic congestion, and nearly 100 cities followed the strategies and promoted the transfer of passengers from cars to public transport. As shown in Figure 1, the passenger volume of bus transit is growing constantly in larger cities in China. In contrast, passenger volume of bus transit in small-medium cities is generally stable as shown in Figure 2. An important reason for the low passenger volume and the low sharing rate of bus transit is the low service quality of this traffic mode in small-medium cities $[1,2]$. Customers' satisfaction is commonly used for measuring service quality in many industries. Furthermore, passengers' satisfaction can inform transportation agencies of their service quality. As such, it is valuable to explore the influencing factors that affect passengers' satisfaction with bus transit in smallmedium cities. It can provide targeted strategies for bus companies to improve the quality of the services as well as to enhance the attraction of bus transit in small-medium cities.

To evaluate the service quality of bus transit, the Ministry of Transport of China proposed the "Index System Assessing Service Quality for Creating Transit Metropolis (ISASCTM)" in 2013 [3]. The evaluation indicators include route setting, bus stop setting, waiting environment, hygienic environment inside the bus, riding comfort, transferring convenience, waiting time, intelligent travel information service, price, and service attitude of the driver [3]. The factors affecting service quality had been explored in 


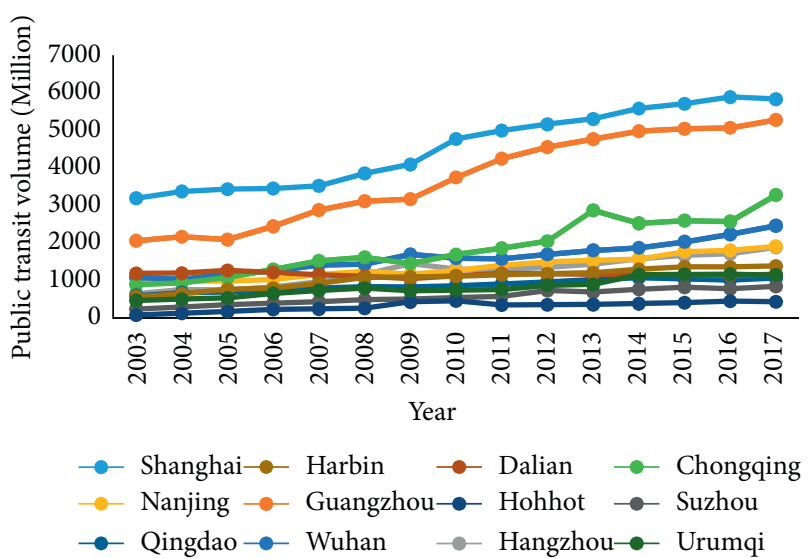

(a)

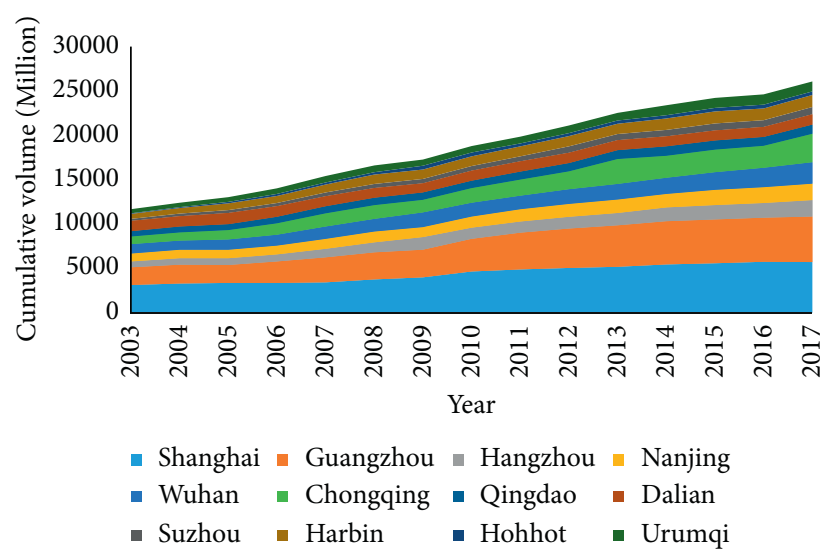

(b)

Figure 1: The passenger volume of public transport in large cities. (a) Passenger volume, (b) cumulative passenger volume.

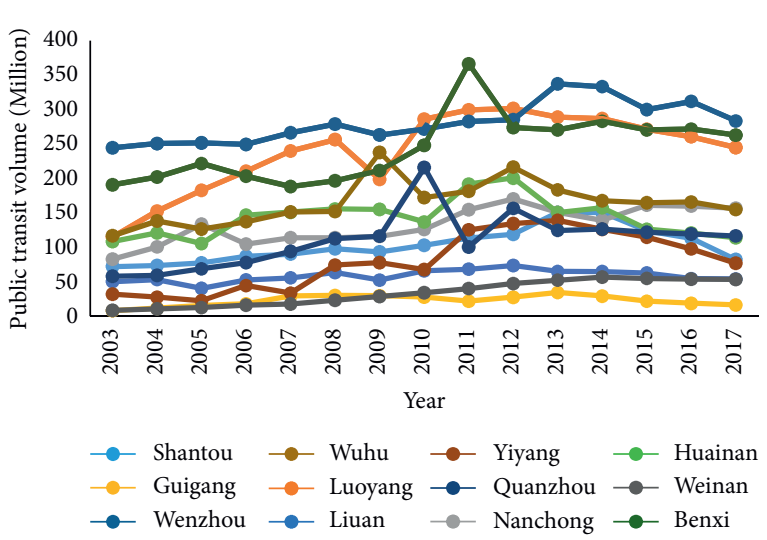

(a)

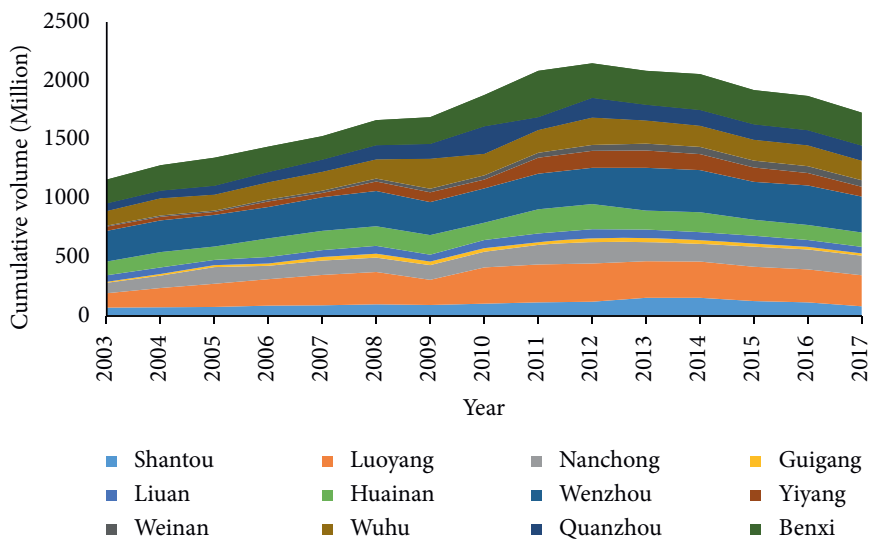

(b)

Figure 2: The passenger volume of public transport in small-medium cities. (a) Passenger volume, (b) cumulative passenger volume.

several existing studies [4-9]. The results from the previous literature provide a reference for the analysis of passengers' satisfaction toward bus transit.

Although a large number of studies have explored the influencing factors of bus satisfaction, these studies mainly focus on the cities in developed countries [10-19] or large cities in developed regions of China [20]. Due to the obvious differences of economic development level, public transit infrastructure development, and residents' subjective satisfaction with public transit between the developed cities and small-medium cities, the existing results from developed cities may not be suitable for guiding the satisfaction improvement of public transit for smallmedium cities in backward areas of China. More importantly, the backward economy in small-medium cities leads to very low investment in public transport infrastructure. At present, how to effectively use every small amount of investment in the urgently improved public transit infrastructure is the key problem for the local governments or bus companies. Accurately identifying the exciting factors affecting passengers' bus satisfaction is the foundation for the local governments to put limited funds into the urgent fields of public transit infrastructure construction.
The objective of this study is to explore the factors affecting the passengers' satisfaction toward bus transit in Chinese small-medium cities. The revealed preference (RP) survey was conducted to collect passengers' data in Weinan city, China. The ordered logit (OL) model and ordered Probit (OP) model were developed to investigate the factors including passengers' attributes, travel activities attributes, and perceived service attributes and may have more applications to other research fields, too [21-24]. The odds ratios (ORs) were used to quantitatively measure the effect of significant variables on passengers' satisfaction. Improving strategies based on the model results were also discussed using the three-factor theory.

\section{Literature Review}

Passengers' satisfaction on bus transit can be collected by online surveys [8], onboard interviews [11, 25-27], and mobile app [28]. Generally, passengers evaluated a specific aspect of bus transit services such as service frequency, reliability, travel time, comfort, and riding stability [17-20, 25-29]. The studies showed that reliable service and 
departure frequency are the most influential attributes associated with passengers' satisfaction toward bus transit [9]. Dell'oli et al. [19] showed that the service quality of bus transit can be enhanced by improving the waiting time, travel comfort, information sources, and departure frequency. Besides, the perceived waiting and travel time were found to be significant factors of passengers' satisfaction [30]. Moreover, punctuality, travel speed, and departure frequency also affected the service quality of bust transit [31].

Besides, several advanced modeling techniques such as structural equation model (SEM), multiple regression, and discrete choice models were used in the analysis of passengers' satisfaction $[1,8,17,25,28,30]$. For example, De Ona et al. [16] applied the SEM to assess the passengers' satisfaction toward the overall service quality of the bus and found that service quality is the most important factor, followed by the personnel and comfort. Then, De Ona et al. [17] also explored the relationship between customers' satisfaction and perceived accessibility of the Subway in Seville, Spain, using SEM models. The results show that service equipment is most important for passengers' satisfaction, followed by accessibility, service availability, information, security, customer service, personal space, and environmental pollution. Ingvardson et al. [30] used a structural equation to investigate the key drivers of satisfaction with public transport and their relationship with travel frequency. Tarigan et al. [31] applied the ordered Probit model to explore the satisfaction of transit passengers on the auxiliary transportation system in Bandung, Indonesia.

What is more, differences in passengers' satisfaction and its influencing factors have been explored for different groups. The transit passengers were classified into six groups and it has been found that the punctuality and security inside the vehicles were the most critical attributes of the perceived service quality [31]. De Oña et al. [27] investigated the perception of different passenger groups regarding the service quality of Milan suburban rail in Italy. They found that although service quality was perceived differently in different populations, the most critical factors were regularity and punctuality. Abenoza et al. [8] identified the most important determinants of satisfaction with bus transit services and investigated the change of the importance of attributes over time among the different passenger groups including inactive travelers, long-distance commuters, urban motorist commuters, rural motorist commuters, and students.

From the above analysis, we can know that the existing researches often take cities in developed countries and cities in developed regions of China as research objects and discuss the difference among different passengers' groups. However, few pieces of research explore the influencing factors of public transit satisfaction in small-medium cities in China and reveal the difference of influencing factors between big cities and small-medium cities. Besides, although the ordered logit model and ordered Probit model are all often used in identifying the significant factors associated with PT satisfaction, the comparison of the ordered logit model and ordered Probit has not been reported in exploring influencing factors of passenger satisfaction toward bus transit. Furthermore, the strategies for improving public transport satisfaction in small-medium cities need to be proposed due to little research.

\section{Data Collection}

3.1. Questionnaire Design. The RP survey was designed and it was conducted in Weinan city. Weinan is a small-medium city located in the eastern part of Guanzhong Plain. By the end of 2018, there were 24 bus routes and 377 bus stops in Weinan [32]. The total length of the bus routes is $187.6 \mathrm{~km}$, and the average length per line is $15.7 \mathrm{~km}$ [32]. The passenger volume reached 50.18 million in 2018 . However, the share rate of bus transit is very low, which is only $13 \%$ in 2018 [32].

The questionnaire in the survey consists of four parts. The first part is the individual attributes of passengers, including gender, age, monthly income, education, occupation, and car ownership. The second part is to investigate the travel activity of passengers, including travel frequency, travel time, and waiting time. The third part is the service quality of bus transit. Ten indicators of service quality of bus transit were selected from ISASCTM, including waiting time, riding comfort, bus route setting, bus stop setting, and so on [3]. The passengers were asked to evaluate each of these service quality attributes based on a 4-point Likert scale from unqualified to excellent. In the last part of the questionnaire, the passengers were asked about their overall satisfaction toward bus transit using a 5-point rating scale from "unsatisfied" to "very satisfied."

3.2. Fields Survey. Face-to-face interviews were conducted on 24 bus routes on May 16, 2019. Sixty junior students from the Transportation Engineering Department of Xi'an University of Architecture and Technology were hired to conduct the interviews. Before the conduction of the survey, the investigators were carefully trained in the lab. The random sampling technique was used in the survey. The investigators were asked to randomly select every fifth person, regardless of gender, age, or other factors. The investigators first explained the purpose of the survey to the respondents and then invited them to participate in the survey. Respondents are guaranteed to participate voluntarily and their responses will be anonymous. Investigators stayed nearby to explain any questions to help the respondents during the survey. The questionnaire would take about 15 minutes to complete. After completing the questionnaire, the respondents were given a small appreciation gift.

3.3. Data Description. 1,397 effective samples were obtained and used for the analysis. As shown in Figure 3, 58.48\% of the passengers are satisfied with the overall service of the bus transit, and $19.18 \%$ of the passengers are very satisfied. Only $0.36 \%$ of the passengers were very dissatisfied with the overall service of the bus transit. The individual attributes are shown in Table 1. Female respondents are slightly more than male. $60 \%$ of the respondents are between the ages of 16 and 45. Regarding education, only a few respondents are 


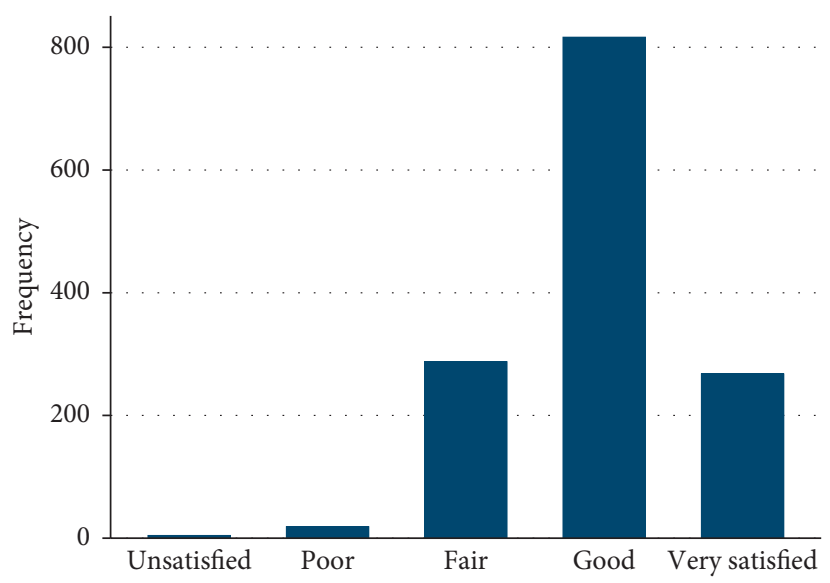

Figure 3: The frequency for passengers' overall satisfaction.

TABLE 1: Respondents' attributes.

\begin{tabular}{|c|c|c|c|c|c|}
\hline Variable & Category & Unit & Code & Frequency & Proportion \\
\hline \multirow{2}{*}{ Gender } & Male & - & 1 & 636 & 45.53 \\
\hline & Female & - & 0 & 761 & 54.47 \\
\hline \multirow{7}{*}{ Age } & $<16$ & Years & 1 & 147 & 10.52 \\
\hline & $16-25$ & Years & 2 & 298 & 21.33 \\
\hline & $26-35$ & Years & 3 & 337 & 24.13 \\
\hline & $36-45$ & Years & 4 & 240 & 17.18 \\
\hline & $46-55$ & Years & 5 & 159 & 11.38 \\
\hline & $56-65$ & Years & 6 & 108 & 7.73 \\
\hline & $>65$ & Years & 7 & 108 & 7.73 \\
\hline \multirow{4}{*}{ Education } & Junior middle school & - & 1 & 404 & 28.92 \\
\hline & High school & - & 2 & 428 & 30.64 \\
\hline & Undergraduate & - & 3 & 537 & 38.44 \\
\hline & Graduate & - & 4 & 28 & 2.00 \\
\hline \multirow{4}{*}{ Occupation } & Government person & - & 1 & 130 & 9.31 \\
\hline & Enterprise staff & - & 2 & 228 & 16.32 \\
\hline & Student & - & 3 & 310 & 22.19 \\
\hline & Self-employed & - & 4 & 729 & 52.18 \\
\hline \multirow{2}{*}{ Cars } & Yes & - & 1 & 690 & 49.39 \\
\hline & No & - & 0 & 707 & 50.61 \\
\hline \multirow{5}{*}{ Monthly income } & $<2000$ & Yuan & 1 & 499 & 35.72 \\
\hline & $2001-4000$ & Yuan & 2 & 534 & 38.22 \\
\hline & $4001-6000$ & Yuan & 3 & 277 & 19.83 \\
\hline & $6001-8000$ & Yuan & 4 & 60 & 4.30 \\
\hline & $>8000$ & Yuan & 5 & 27 & 1.93 \\
\hline
\end{tabular}

graduate students, and the other education levels are balanced. The proportion of self-employed is the highest, followed by the students and enterprise staff. Almost half of the respondents own cars, although the monthly income of $73.94 \%$ of respondents is below 4,000 Yuan.

The respondent' travel activity attributes are shown in Table 2. Nearly half of the respondents take bus transit twice a day, while few respondents take bus transit three or more times a day. The majority of respondents spend less than 60 minutes a day by bus transit, and only a few respondents spend more than 60 minutes. The waiting time is less than 15 minutes for the majority of the respondents. Table 3 shows the satisfaction level for each service attribute. It is shown that more than $80 \%$ of respondents consider the service quality attributes of bus transit to be "well" and "excellent," while a small proportion evaluate these attributes as "unqualified."

\section{Methodology}

4.1. Ordered Logit Model. The ordered logit (OL) model is derived by defining an unobserved variable $Z$, which is used as a basis for modeling the ordinal data. In this study, passengers' satisfaction is an ordinal variable that consists of five levels: 1, unsatisfied; 2, poor; 3, fair; 4, good; and 5, very satisfied. The general specification of the ordinal variable for each observation is $[33,34]$

$$
Z=\boldsymbol{\beta} \mathbf{X}+\varepsilon_{i}
$$


TABLE 2: Respondents' travel activity attributes.

\begin{tabular}{|c|c|c|c|c|c|}
\hline Variable & Category & Unit & Code & Frequency & Proportion \\
\hline \multirow{2}{*}{ Travel purposes } & Mandatory travel & - & 1 & 742 & 53.10 \\
\hline & Leisure travel & - & 0 & 655 & 46.90 \\
\hline \multirow{6}{*}{ Traveling frequency } & 0 & Times/day & 1 & 373 & 26.70 \\
\hline & 1 & Times/day & 2 & 267 & 19.11 \\
\hline & 2 & Times/day & 3 & 564 & 49.37 \\
\hline & 3 & Times/day & 4 & 83 & 5.94 \\
\hline & 4 & Times/day & 5 & 84 & 6.02 \\
\hline & 5 & Times/day & 6 & 26 & 1.86 \\
\hline \multirow{5}{*}{ Daily average traveling time } & $0-30$ & Minutes & 1 & 819 & 58.63 \\
\hline & $30-60$ & Minutes & 2 & 489 & 35.00 \\
\hline & $60-90$ & Minutes & 3 & 67 & 4.80 \\
\hline & $90-120$ & Minutes & 4 & 16 & 1.15 \\
\hline & $>120$ & Minutes & 5 & 6 & 0.42 \\
\hline \multirow{5}{*}{ Daily average waiting time } & $0-5$ & Minutes & 1 & 423 & 30.28 \\
\hline & $5-10$ & Minutes & 2 & 551 & 39.44 \\
\hline & $10-15$ & Minutes & 3 & 289 & 20.69 \\
\hline & $15-20$ & Minutes & 4 & 87 & 6.23 \\
\hline & $>20$ & Minutes & 5 & 47 & 3.36 \\
\hline
\end{tabular}

TABLE 3: Respondents' service quality perception attributes.

\begin{tabular}{|c|c|c|c|c|}
\hline Variable & Category & Code & Frequency & Proportion (\%) \\
\hline \multirow{4}{*}{ Hygienic environment inside the bus } & Unqualified & 1 & 8 & 0.57 \\
\hline & Qualified & 2 & 162 & 11.60 \\
\hline & Well & 3 & 605 & 43.31 \\
\hline & Excellent & 4 & 622 & 44.52 \\
\hline \multirow{4}{*}{ Service attitude of the driver } & Unqualified & 1 & 31 & 2.22 \\
\hline & Qualified & 2 & 156 & 11.17 \\
\hline & Well & 3 & 613 & 43.88 \\
\hline & Excellent & 4 & 597 & 42.73 \\
\hline \multirow{4}{*}{ Riding comfort } & Unqualified & 1 & 18 & 1.29 \\
\hline & Qualified & 2 & 174 & 12.46 \\
\hline & Well & 3 & 657 & 47.03 \\
\hline & Excellent & 4 & 548 & 39.22 \\
\hline \multirow{4}{*}{ Intelligent travel information service } & Unqualified & 1 & 16 & 1.15 \\
\hline & Qualified & 2 & 192 & 13.74 \\
\hline & Well & 3 & 633 & 45.31 \\
\hline & Excellent & 4 & 556 & 39.80 \\
\hline \multirow{4}{*}{ Transferring convenience } & Unqualified & 1 & 17 & 1.22 \\
\hline & Qualified & 2 & 154 & 11.03 \\
\hline & Well & 3 & 711 & 50.89 \\
\hline & Excellent & 4 & 515 & 36.86 \\
\hline \multirow{4}{*}{ Bus route setting } & Unqualified & 1 & 30 & 2.15 \\
\hline & Qualified & 2 & 185 & 13.24 \\
\hline & Well & 3 & 626 & 44.81 \\
\hline & Excellent & 4 & 556 & 39.80 \\
\hline \multirow{4}{*}{ Bus stop setting } & Unqualified & 1 & 24 & 1.72 \\
\hline & Qualified & 2 & 196 & 14.03 \\
\hline & Well & 3 & 627 & 44.88 \\
\hline & Excellent & 4 & 550 & 39.37 \\
\hline \multirow{4}{*}{ Waiting environment } & Unqualified & 1 & 19 & 1.36 \\
\hline & Qualified & 2 & 202 & 14.46 \\
\hline & Well & 3 & 647 & 46.31 \\
\hline & Excellent & 4 & 529 & 37.87 \\
\hline \multirow{4}{*}{ Perceived waiting time } & Unqualified & 1 & 63 & 4.51 \\
\hline & Qualified & 2 & 171 & 12.24 \\
\hline & Well & 3 & 693 & 49.61 \\
\hline & Excellent & 4 & 470 & 33.64 \\
\hline \multirow{4}{*}{ Ticket price } & Unqualified & 1 & 62 & 4.44 \\
\hline & Qualified & 2 & 254 & 18.18 \\
\hline & Well & 3 & 548 & 39.23 \\
\hline & Excellent & 4 & 533 & 38.15 \\
\hline
\end{tabular}


where $\mathbf{X}$ is a vector of explanatory variables determining the discrete ordering $i$ for each observation, $\boldsymbol{\beta}$ is a vector of coefficients associated with the explanatory variables, and $\varepsilon_{i}$ is a random error term. Using the above equation, observed passengers' satisfaction $y$ can be defined as $[33,34]$

$$
y= \begin{cases}1 & \text { if } Z \leq \mu_{1} \text { (Unsatisfied), } \\ 2 & \text { if } \mu_{1}<Z \leq \mu_{2} \text { (Poor), } \\ 3 & \text { if } \mu_{2}<Z \leq \mu_{3} \text { (Fair), } \\ 4 & \text { if } \mu_{3}<Z \leq \mu_{4} \text { (Good), } \\ 5 & \text { if } Z>\mu_{4} \text { (Very satisfied), }\end{cases}
$$

where $u_{i}$ is unknown estimable parameters (also referred to as thresholds) that define the passengers' satisfaction $y$ and corresponds to integer order.

To estimate the parameters $u_{i}$ with the model parameters $\beta$, the random error term is assumed to be independent and identically distributed with the logistic distribution. Based on the assumption, an ordered logit model can be derived. The probability that a passenger's satisfaction belongs to either of the five levels is defined as

$$
P y=i=\Omega\left(u_{i}-\boldsymbol{\beta} \mathbf{X}\right)-\Omega\left(u_{i-1}-\boldsymbol{\beta} \mathbf{X}\right),
$$

where $i=1,2,3,4,5 ; \Omega(\bullet)$ is the standard logistic cumulative distribution function $u_{i}$ and $u_{i-1}$ represent the upper and lower thresholds for outcome $i$.

The parameters can be estimated using the maximum likelihood estimation approach. For a population of $N$ observations, the log-likelihood function of the OL model is

$$
L L=\sum_{n=1}^{N} \sum_{i=1}^{I} \delta_{i n} \ln \left[\Omega u_{i}-\boldsymbol{\beta} \mathbf{X}_{\mathbf{n}}-\Omega\left(u_{i-1}-\boldsymbol{\beta} \mathbf{X}_{\mathbf{n}}\right)\right],
$$

where $\delta_{\text {in }}$ is equal to 1 if the observed discrete outcome for observation $n$ is $i$, and zero otherwise.

4.2. Ordered Probit Model. The ordered Probit (OP) model has also been widely used for fitting the data structure of an ordinal response $[10,35]$. Assuming that $Y$ represents the passengers' satisfaction, then a latent variable $Y^{*}$ is given as

$$
Y^{*}=\mathbf{X} \boldsymbol{\beta}^{\prime}+\varepsilon^{\prime},
$$

where $\mathbf{X}$ is the vector of explanatory variables, $\beta^{\prime}$ is the vector of coefficients associated with the explanatory variables, and $\varepsilon^{\prime}$ is a random error term following a standard normal distribution. The value of the dependent variable $\mathbf{Y}$ is then given as [33]

$$
Y= \begin{cases}1 & \text { if } Y^{*} \leq \tau_{1} \\ j & \text { if } \tau_{j-1} \leq \tau_{j} \\ J & \text { if } \tau_{J-1} \leq Y^{*}\end{cases}
$$

where $J$ is the number of passengers' satisfaction levels $(J=5)$ and $\tau_{j}$ is the threshold parameter (cut-off points) to be estimated. The probability for $Y$ taking a particular value $j$ is given by

$$
\begin{aligned}
P(Y=1) & =\Phi\left(\tau_{1}-\mathbf{X} \boldsymbol{\beta}^{\prime}\right) \\
P(Y=j) & =\Phi\left(\tau_{j}-\mathbf{X} \boldsymbol{\beta}^{\prime}\right)-\Phi\left(\tau_{j-1}-\mathbf{X} \boldsymbol{\beta}^{\prime}\right) \\
P(Y=J) & =1-\Phi\left(\tau_{J-1}-\mathbf{X} \boldsymbol{\beta}^{\prime}\right)
\end{aligned}
$$

where $P(\mathbf{Y}=j)$ is the probability of response variable taking a specific level $j, \Phi(\bullet)$ is the standard normal cumulative distribution function, and the threshold parameter $\tau_{j}$ satisfies the restriction $\tau_{1} \cdots<\tau_{j}<\cdots<\tau_{J-1}$.

The parameters of $\beta^{\prime}$ can be determined by the maximum likelihood estimation method $[10,33,35]$. The likelihood function is given as

$$
L=\prod_{n=1}^{N} \prod_{j=1}^{J}\left[\Phi \tau_{j}-\boldsymbol{\beta}^{\prime} \mathbf{X}_{\mathbf{n}}-\Phi \tau_{j-1}-\boldsymbol{\beta}^{\prime} \mathbf{X}_{\mathbf{n}}\right]^{\delta_{j n}}
$$

where $N$ is the number of explanatory variables and $\delta_{j n}$ is equal to 1 if the observed discrete outcome for observation $n$ is $j$, and zero otherwise.

4.3. Model Comparison Measures. The Akaike information criterion (AIC) and Bayesian information criterion (BIC) were used to compare the goodness-of-fit of the models [33]. The AIC is given as

$$
\mathrm{AIC}=2 K-2 \ln L
$$

where $K$ represents the number of model parameters including intercept term and explanatory variable that need to be estimated for the model and $L$ is the maximum likelihood.

$\mathrm{BIC}$ is closely related to AIC and has a more obvious penalty effect on free variables [33]. The BIC is given as

$$
\mathrm{BIC}=\mathrm{AIC}+K(\ln N-2),
$$

where $N$ represents the number of samples. The smaller the $\mathrm{AIC}$ and BIC, the higher the goodness of fitting.

4.4. Odds Ratio. The odds ratio (OR) was used to quantify the impacts of the explanatory variables on the outcome. The OR was calculated for variables of interest in the ordered logit regressions. The OR of an explanatory variable represents the increase in the odds of the outcome if the value of the variable increases by one unit [33, 36-39]. The OR for a variable $x_{m}$ can be calculated as

$$
\mathrm{OR}=\frac{\operatorname{odds}\left(\mathrm{X}, x_{m+1}\right)}{\operatorname{odds}\left(\mathrm{X}, x_{m}\right)}=\frac{\exp (\mathbf{X} \boldsymbol{\beta}) \times \exp \left(\beta_{m}\right)}{\exp (\mathbf{X} \boldsymbol{\beta})}=\exp \left(\beta_{m}\right)
$$

\section{Results and Discussion}

5.1. Model Results. The multicollinearity test was conducted before modeling and the variance inflation factor (VIF) of each independent variable is less than 5 (see Table 4), indicating that there is no serious multicollinearity between the independent variables [40]. Then, the OL and OP models were developed to explore the significant variables associated with passengers' satisfaction. The software STATA 15.0 
TABLE 4: Collinearity results of independent variables.

\begin{tabular}{ll}
\hline Variables & VIF \\
\hline Education & 1.46 \\
Occupation & 1.38 \\
Monthly income & 1.32 \\
Traveling frequency & 1.32 \\
Age & 1.30 \\
Travel purposes & 1.28 \\
Daily average traveling time & 1.24 \\
Daily average waiting time & 1.21 \\
Car ownership & 1.09 \\
Gender & 1.06 \\
Bus route setting & 2.43 \\
Bus stop setting & 2.35 \\
Waiting environment & 2.29 \\
Hygienic environment inside the bus & 2.11 \\
Riding comfort & 2.06 \\
Transferring convenience & 1.93 \\
Perceived waiting time & 1.75 \\
Intelligent travel information service & 1.74 \\
Ticket price & 1.63 \\
Service attitude of the driver & 1.60 \\
\hline
\end{tabular}

was used as the modeling platform for model estimation. The estimation results of the OL and OP models are given in Table 5.

As shown in Table 5, ten variables in the OL model including age, daily average waiting time, perceived waiting time, transferring convenience, service attitude of the driver, intelligent travel information service, hygienic environment inside the bus, ticket price, bus route setting, and bus stop setting were found to be significant at the $90 \%$ confidence level. For the OP model, gender, age, daily waiting time, perceived waiting time, transferring convenience, service attitude of the driver, intelligent travel information service, waiting environment, hygienic environment inside the bus, ticket price, and bus stop setting had significant effect on passengers' satisfaction toward bus transit at the 90\% confidence level. It is also found that both the AIC and BIC of the OL model were smaller than those of the OP model, indicating that the OL model outperforms the OP model. Therefore, the estimation results of the OL model were selected for analysis.

5.2. Interpretation of Model Results. The ORs were used to quantitatively measure the effect of significant factors on passengers' satisfaction toward bus transit. The ORs based on the OL model results are shown in Table 5. The passengers' satisfaction toward bus transit increased with their age. According to the OR analysis, the odds of an additional level of passengers' satisfaction toward bus transit would increase by $10.29 \%$ for every 10 years of age increased. The possible reason is that older passengers have lower service demand and are more likely to be satisfied with the bus service [25].

Passengers' satisfaction was significantly associated with the daily average waiting time. The negative sign of daily average waiting time indicates that an increase in this variable is associated with a decrease in passengers' satisfaction level. The OR of daily waiting time is 0.889 , showing that the odds of an additional level of passengers' satisfaction would reduce by $11.1 \%$ with the increase of daily average waiting time of $5 \mathrm{~min}$. This finding is intuitive that longer waiting time will cause a lower satisfaction of passengers. This finding is supported by the results of a previous study [26].

The variable of waiting time is service quality perception attributes. It was found that this variable has a positive effect on passengers' satisfaction toward bus transit, which indicated that the more satisfied passengers are with the wait time, the more satisfied they are with the bus service. According to the OR result, the odds of an additional level of passengers' satisfaction would increase by $18.71 \%$ for each additional level of the perceptive waiting time. This result is reasonable that the high reliability of the bus makes passengers more satisfied with the bus waiting time, which leads to a higher satisfaction level of passengers by bus transit. This finding is in line with the results of previous studies that reliability is positively associated with the passengers' satisfaction toward bus transit [18].

Transferring convenience has a significantly positive influence on passengers' satisfaction. Based on the OR result, the odds of an additional level of passengers' satisfaction would increase by $36.02 \%$ for each additional level of the transferring convenience. This result is straightforward that improving transfer efficiency can promote the passengers' satisfaction toward bus transit. The finding is consistent with the results of previous studies [2] that "transferring is easy" is positively associated with passengers' satisfaction with public transport.

The service attitude of the driver was found to be significantly related to passengers' satisfaction. According to the OR result, the odds of an additional level of passengers' satisfaction would increase by $31.71 \%$ for each additional level of the service attitude of the driver. This result is intuitive that the good service attitude of the driver can make passengers feel more warm and comfortable, resulting in higher satisfaction of passengers. The finding is supported by previous studies that the "personnel attitude" or "driver's manner" was positively associated with passengers' satisfaction with public transport [2].

The intelligent travel information service has a significantly positive influence on passengers' satisfaction. The intelligent travel information service can provide passengers with much useful information such as bus location, travel schedule, and transfer information. Based on the OR result, the odds of an additional level of passengers' satisfaction would increase by $45.06 \%$ for each additional level of the intelligent travel information service. This result is straightforward that improving the intelligent travel information service can help passengers get the operation and dispatching information of the bus, which can hoist passengers' satisfaction. The finding is consistent with previous studies by Börjesson et al. [12] that the "information at the stop" or "information personnel" is positively associated with passengers' satisfaction with public transport. 
TABle 5: Estimation results of the OL model and OP model.

\begin{tabular}{|c|c|c|c|c|c|}
\hline \multirow{2}{*}{ Variables } & \multicolumn{2}{|c|}{$\mathrm{OL}$} & \multicolumn{2}{|c|}{ OP } & \multirow{2}{*}{$\mathrm{OR}^{\#}$} \\
\hline & Coef. & $p$ value & Coef. & $p$ value & \\
\hline \multicolumn{6}{|l|}{ Individual attributes } \\
\hline Gender Male vs female & -0.1905 & 0.1090 & -0.1192 & $0.0770^{*}$ & \\
\hline Cars Yes vs no & 0.0558 & 0.6330 & 0.0048 & 0.9420 & \\
\hline Age & 0.0979 & $0.0390^{* *}$ & 0.0467 & $0.0820^{*}$ & 1.1029 \\
\hline \multicolumn{6}{|l|}{ Education } \\
\hline High vs junior & 0.2096 & 0.1710 & 0.0932 & 0.2830 & \\
\hline Undergraduate vs junior & 0.0513 & 0.7570 & 0.0000 & 0.9990 & \\
\hline Graduate vs junior & -0.1904 & 0.6540 & -0.1853 & 0.4410 & \\
\hline \multicolumn{6}{|l|}{ Occupation } \\
\hline Enterprise staff vs government person & -0.0653 & 0.7800 & -0.0458 & 0.7280 & \\
\hline Student vs government person & 0.0391 & 0.8880 & -0.0254 & 0.8710 & \\
\hline Self-employed vs government person & -0.1585 & 0.6540 & -0.0795 & 0.5270 & \\
\hline Monthly income & 0.0258 & 0.7340 & 0.0174 & 0.6910 & \\
\hline Travel purposes (mandatory travel vs leisure travel) & -0.1230 & 0.3330 & -0.0555 & 0.4390 & \\
\hline \multicolumn{6}{|l|}{ Travel activity attributes } \\
\hline Traveling frequency & 0.0144 & 0.7830 & 0.0086 & 0.7730 & \\
\hline Daily average traveling time & -0.1316 & 0.1450 & -0.0793 & 0.1260 & \\
\hline Daily average waiting time & -0.1177 & $0.0490^{* *}$ & -0.0608 & $0.0740^{*}$ & 0.8890 \\
\hline \multicolumn{6}{|l|}{ Perceived service attributes } \\
\hline Perceived waiting time & 0.1715 & $0.0690^{*}$ & 0.1161 & $0.0270^{* *}$ & 1.1871 \\
\hline Transferring convenience & 0.3076 & $0.0070^{* *}$ & 0.1485 & $0.0190^{* *}$ & 1.3602 \\
\hline Service attitude of the driver & 0.2754 & $0.0040^{* *}$ & 0.1396 & $0.0090^{* *}$ & 1.3171 \\
\hline Intelligent travel information service & 0.3720 & $0.0001^{* * *}$ & 0.1957 & $0.0010^{* * *}$ & 1.4506 \\
\hline Riding comfort & 0.0244 & 0.8280 & 0.0038 & 0.9520 & \\
\hline Waiting environment & 0.1754 & 0.1320 & 0.1195 & $0.0660^{*}$ & \\
\hline Hygienic environment inside the bus & 0.4006 & $0.0010^{* * *}$ & 0.1832 & $0.0060^{* *}$ & 1.4927 \\
\hline Ticket price & 0.3768 & $0.0001^{* * *}$ & 0.2101 & $0.0001^{* * *}$ & 1.4576 \\
\hline Bus route setting & 0.2022 & $0.0910^{*}$ & 0.0882 & 0.1730 & 1.2241 \\
\hline Bus stop setting & 0.5875 & $0.0001^{* * *}$ & 0.3348 & $0.0001^{* * *}$ & 1.7995 \\
\hline \multicolumn{6}{|l|}{ Thresholds } \\
\hline Unqualified & 2.1488 & & 1.3401 & & \\
\hline Poor & 3.8288 & & 1.9989 & & \\
\hline Fair & 7.2371 & & 3.7469 & & \\
\hline Good & 11.1399 & & 5.9293 & & \\
\hline \multicolumn{6}{|l|}{ Model measures } \\
\hline AIC & 2324.5520 & & 2346.7360 & & \\
\hline BIC & 2471.3310 & & 2493.5140 & & \\
\hline
\end{tabular}

${ }^{\#}$ ORs of the variables in the OL model, ${ }^{*}$ variables that are significant at $90 \%$ confidence level, ${ }^{* *}$ variables that are significant at $95 \%$ confidence level, ${ }^{* * *}$ variables that are significant at $99 \%$ confidence level.

The hygienic environment inside the bus was found to be a significantly positive association with passengers' satisfaction. According to the OR result, the odds of an additional level of passengers' satisfaction would increase by $49.27 \%$ for each additional level of the hygienic environment inside the bus. This result is reasonable that the hygienic environment inside the bus can make passengers feel the fresh air, ensure their health, and give passengers a better travel experience. As stated by Mouwen [41], the "vehicle tidiness" is positively associated with passengers' satisfaction with public transport. Improving the hygienic environment can promote the passengers' satisfaction.

The ticket price had a significantly positive influence on passengers' satisfaction. Based on the OR result, the odds of an additional level of passengers' satisfaction toward the bus transit would increase by $45.76 \%$ for each additional satisfaction level of passengers toward the ticket price. Although some studies reported that bus ticket price has no impact on passengers' satisfaction $[2,12]$, this study indicates that price had a significant effect on passengers' satisfaction toward bus transit. This result can be explained that improving the pricing system such as variable fare can improve the passengers' satisfaction.

The results had shown that the bus route setting had a significantly positive effect on passengers' satisfaction. According to the OR result, the odds of an additional level of passengers' satisfaction would increase by $22.41 \%$ for each additional level of bus route setting. The finding is straightforward because improving route settings of the bus network can reduce the non-linear coefficient of the bus line and improve passenger efficiency, which is helpful to raise passengers' satisfaction.

The bus stop setting was found to have a significantly positive influence on passengers' satisfaction. Based on the OR result, the odds of an additional level of passengers' satisfaction would increase by $79.95 \%$ for each additional 


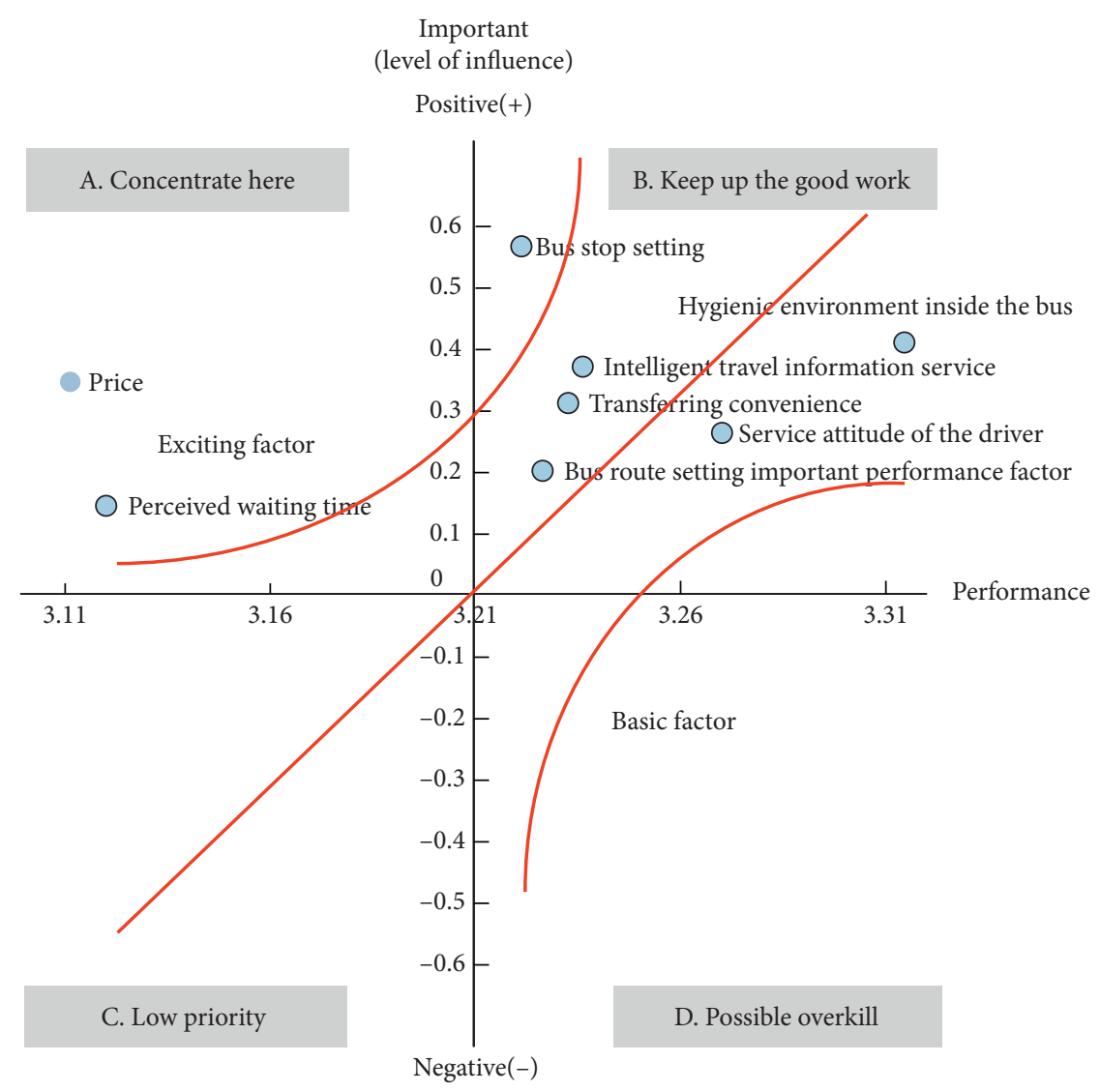

FIgURE 4: IPA matrices of passengers' satisfaction toward bus transit.

level of bus stop setting. This result is intuitive because improving the service quality of the stop setting can effectively shorten the walking distance of passengers, which can promote passenger satisfaction toward bus transit. The finding is inconsistent with the results of previous studies $[8,26]$ where the "stop setting" was found not to be significantly associated with the passengers' satisfaction toward public transit.

5.3. Improving Strategies. Importance performance analysis (IPA) has been widely used to investigate service improvement priorities based on customer satisfaction surveys. As pointed out by Cao et al. [1], three factors including basic factors, performance factors, and exciting factors can be identified using the three-factor theory of IPA. Basic factors refer to these attributes which have significant impacts on passengers' satisfaction when they perform poorly. However, when they perform well, they do not increase passengers' satisfaction. Performance factors refer to these attributes which have significant impacts when they perform both well and poorly or have a linear and symmetric relationship with passengers' satisfaction, as assumed in the IPA. Exciting factors refer to these attributes which have significant impacts when they perform well or increase passengers' satisfaction if they are well delivered. However, when they do not perform well, they do not adversely affect passengers' satisfaction.
As shown in Figure 4, the factor structure of bus transit attributes was identified by using the three-factor theory. Bus ticket price, perceived waiting time, bus stop setting, intelligent travel information service, transferring convenience, and bus route setting were categorized as exciting factors. Particularly, bus ticket price and perceived waiting time should be concentrated on by bus transit companies and the government because of their low performance. Besides, the hygienic environment inside the bus and service attitude of the driver were categorized as important performance factors that need to keep up the good work. According to the result of the three-factor analysis, it is recommended that the exciting factors should be given priority and the important performance factors should be defined as a second priority to improve the passengers' satisfaction toward bus transit as well as enhance the service quality of bus transit.

Therefore, the construction of bus dedicated lanes that are conducive to improving the reliability of bus transit and reducing waiting time is an efficient measure to improve passengers' satisfaction. Secondly, the dynamic charging system is an important countermeasure to optimize the bus ticket price for different seasons, different zones, and different age groups. Also, the optimization of bus routes and stop setting, building bus information systems, and providing travel information service could improve the service quality of bus transit and promote passengers' satisfaction. 


\section{Conclusion}

This study investigated the influencing factors of passengers' satisfaction toward bus transit and developed countermeasures to improve the bus transit service quality and promote passengers' satisfaction. Data were collected by an RP survey in a small-medium city, Weinan city, China. The OL model and OP model were developed to explore the significant factors of passengers' satisfaction toward bus transit. The OR technique was used to quantitatively measure the effect of the significant variables. Improving strategies of passengers' satisfaction were proposed based on the model results and the three-factor theory analysis.

The comparison result showed that the OL model outperforms the OP model. The factors that significantly affect passengers' satisfaction were bus stop setting, ticket price, intelligent travel information service, hygienic environment inside the bus, service attitude of the driver, transferring convenience, perceived waiting time, and bus route setting. The three-factor analysis showed that ticket price, perceived waiting time, bus stop setting, intelligent travel information service, transferring convenience, and bus route setting were exciting factors. The hygienic environment inside the bus and service attitude of the driver were important performance factors. Countermeasures including the construction of bus dedicated lanes, dynamic charging system, optimizing bus routes and stop setting, and providing travel information service were proposed and discussed.

There are some limitations to this study. The RP survey is conducted among the bus transit passengers. Future studies should expand the current RP survey to non-bus users, and more potential factors of passenger's satisfaction could be explored. Moreover, the comparison of passengers' satisfaction toward bus transit should be conducted between larger cities and small-medium cities to reveal the different influencing factors. Furthermore, the state-of-the-art estimation approaches such as the full Bayesian estimation method should be applied in the OL and OP models.

\section{Data Availability}

The data used to support the findings of this study are available from the corresponding author upon request.

\section{Conflicts of Interest}

The authors declare that they have no conflicts of interest.

\section{References}

[1] J. Cao and X. Cao, "Comparing importance-performance analysis and three-factor theory in assessing rider satisfaction with transit," Journal of Transport and Land Use, vol. 10, no. 1, pp. 65-68, 2017.

[2] X. Wu, J. Cao, and J. Huting, "Using three-factor theory to identify improvement priorities for express and local bus services: an application of regression with dummy variables in the Twin Cities," Transportation Research Part A: Policy and Practice, vol. 113, pp. 184-196, 2018.
[3] Ministry of Transport of China, Bus Urban Assessment and Evaluation Index System (Ministry of Transport of China Publication No. 387), Government Printing Office, Beijing, 2013.

[4] L. Eboli and G. Mazzulla, "Relationships between rail passengers' satisfaction and service quality: a framework for identifying key service factors," Public Transport, vol. 7, no. 2, pp. 185-201, 2015.

[5] B. Guirao, A. García-Pastor, and M. E. López-Lambas, "The importance of service quality attributes in public transportation: narrowing the gap between scientific research and practitioners' needs," Transport Policy, vol. 49, pp. 68-77, 2016.

[6] D. Wan, C. Kamga, J. Liu, A. Sugiura, and E. B. Beaton, "Rider perception of a "light" bus rapid transit system-the New York city select bus service," Transport Policy, vol. 49, pp. 41-55, 2016.

[7] J. Soza-Parra, S. Raveau, J. C. Muñoz, and O. Cats, "The underlying effect of public transport reliability on users' satisfaction," Transportation Research Part A: Policy and Practice, vol. 126, pp. 83-93, 2019.

[8] R. F. Abenoza, O. Cats, and Y. O. Susilo, "Travel satisfaction with public transport: determinants, user classes, regional disparities and their evolution," Transportation Research Part A: Policy and Practice, vol. 95, pp. 64-84, 2017.

[9] J. Allen, L. Eboli, G. Mazzulla, and J. D. Ortúzar, "Effect of critical incidents on public transport satisfaction and loyalty: an ordinal probit SEM-MIMIC approach," Transportation, vol. 47, pp. 827-863, 2018.

[10] F. Alemi, G. Circella, P. Mokhtarian, and S. Handy, "What drives the use of ridehailing in California? ordered probit models of the usage frequency of Uber and Lyft," Transportation Research Part C: Emerging Technologies:Emerging Technologies, vol. 102, pp. 233-248, 2019.

[11] M. J. Beck and J. M. Rose, "The best of times and the worst of times: a new best-worst measure of attitudes toward public transport experiences," Transportation Research Part A: Policy and Practice, vol. 86, pp. 108-123, 2016.

[12] M. Börjesson and I. Rubensson, "Satisfaction with crowding and other attributes in public transport," Transport Policy, vol. 79, pp. 213-222, 2019.

[13] R. Concepción, J. C. Martín, and E. Raquel, "Using stated preferences to analyze the service quality of public transport," International Journal of Sustainable Transportation, vol. 8, pp. 28-46, 2014.

[14] V. Dea and A. El-Geneidy, "Enjoying loyalty: the relationship between service quality; customer satisfaction; and behavioral intentions in PT," Research in Transportation Economics, vol. 59, pp. 50-59, 2016.

[15] E. Dimitrios, A. Constantinos, T. Yannis, and S. Eleana, "Factors affecting bus users' satisfaction in times of economic crisis," Transportation Research Part A: Policy and Practice, vol. 114, pp. 3-12, 2018.

[16] J. D. Ona, R. D. Ona, L. Eboli, and G. Mazzulla, "Perceived service quality in bus transit service: a structural equation approach," Transport Policy, vol. 29, pp. 219-226, 2013.

[17] R. D. Ona, J. L. Machad, and J. D. Ona, "Perceived service quality; customer satisfaction; and behavioral intentions: structural equation model for the metro of Seville; Spain," Transportation Research Record, vol. 2538, no. 1, pp. 76-85, 2015.

[18] J. d. Ona, R. d. Ona, and C. Garrido, "Extraction of attribute importance from satisfaction surveys with data mining 
techniques: a comparison between neural networks and decision trees," Transportation Letters, vol. 9, pp. 39-48, 2017.

[19] L. Dell'Olio, I. Angel, and C. Patricia, "The quality of service desired by public transport users," Transport Policy, vol. 18, pp. 217-227, 2011.

[20] C. Zhang, X. Cao, A. Nagpure et al., "Exploring rider satisfaction with transit service in Indore; India: an application of the three-factor theory," Transportation Letters, vol. 11, no. 8, pp. 1-9, 2017.

[21] R. M. S. Costa, T. van Andel, P. Pavone, and S. Pulvirenti, "The pre-Linnaean herbarium of Paolo Boccone (1633-1704) kept in Leiden (The Netherlands) and its connections with the imprinted one in Paris," Plant Biosystems, vol. 3, no. 152, pp. 489-500, 2018.

[22] G. Ferrauto, R. M. S. Costa, P. Pavone, and G. L. Cantarella, "Human impact assessment on the Sicilian agroecosystems through the evaluation of melliferous areas," Annali Di Botanica, vol. 3, pp. 237-244, 2013.

[23] J. Tao, A. Hassan, C. Qibing et al., "Psychological and physiological relaxation induced by nature-working with ornamental plants," Discrete Dynamics in Nature and Society, vol. 2020, Article ID 6784512, 7 pages, 2020.

[24] Z. Tang, X. Liu, Y. Wang, and D. Ma, "Integrated inventorytransportation scheduling with sustainability-dependent demand under carbon emission policies," Discrete Dynamics in Nature and Society, vol. 2020, Article ID 2510413, 15 pages, 2020.

[25] C. Zhang, Z. Juan, W. Lu, and G. Xiao, "Do the organizational forms affect passenger satisfaction? evidence from Chinese public transport service," Transportation Research Part A: Policy and Practice, vol. 94, pp. 129-148, 2016.

[26] E. Echaniz, C. Q. Ho, A. Rodriguez, and L. dell'Olio, "Comparing best-worst and ordered logit approaches for user satisfaction in transit services," Transportation Research Part A: Policy and Practice, vol. 130, pp. 752-769, 2019.

[27] J. de Oña, R. de Oña, L. Eboli, and G. Mazzulla, "Heterogeneity in perceptions of service quality among groups of railway passengers," International Journal of Sustainable Transportation, vol. 9, no. 8, pp. 612-626, 2015.

[28] M. Fellesson and M. Friman, "Perceived satisfaction with public transport service in nine European cities," Journal of the Transportation Research Forum, vol. 47, no. 3, pp. 93-103, 2007.

[29] B. Guirao, A. García, A. García, M. López, C. Acha, and J. Comendador, "New QR survey methodologies to analyze user perception of service quality in public transport: the experience of Madrid," Journal of Public Transportation, vol. 18, no. 3, pp. 71-88, 2015.

[30] J. B. Ingvardson and O. A. Nielsen, "The relationship between norms, satisfaction and public transport use: a comparison across six European cities using structural equation modelling," Transportation Research Part A: Policy and Practice, vol. 126, pp. 37-57, 2019.

[31] A. K. M. Tarigan, Y. O. Susilo, and T. B. Joewono, "Segmentation of paratransit users based on service quality and travel behaviour in Bandung, Indonesia," Transportation Planning and Technology, vol. 37, no. 2, pp. 200-218, 2014.

[32] Weinan Bus Company \& Xi' an University of Architecture and Technology, Bus survey report of Weinan City, Wei'nan, China, 2018.

[33] S. Washington, M. Karlaftis, and F. Mannering, Statistical and Econometric Methods for Transportation Data Analysis, Chapman \& Hall/CRC, Florida, BR, USA, 2003.
[34] M. Rezapour, M. Moomen, and K. Ksaibati, "Ordered logistic models of influencing factors on crash injury severity of single and multiple-vehicle downgrade crashes: a case study in Wyoming," Journal of Safety Research, vol. 68, pp. 107-118, 2019.

[35] S. Hassen, "The effect of farmyard manure on the continued and discontinued use of inorganic fertilizer in Ethiopia: an ordered probit analysis," Land Use Policy, vol. 72, pp. 523532, 2018.

[36] Y. Guo, Z. Li, Y. Wu, and C. Xu, "Exploring unobserved heterogeneity in bicyclists' red-light running behaviors at different crossing facilities," Accident Analysis \& Prevention, vol. 115, pp. 118-127, 2018.

[37] Y. Guo, Z. Li, Y. Wu, and C. Xu, "Evaluating factors affecting electric bike users' registration of license plate in China using Bayesian approach," Transportation Research Part F: Traffic Psychology and Behaviour, vol. 59, pp. 212-221, 2018.

[38] X. Li, J. Tang, X. Hu, and W. Wang, "Assessing intercity multimodal choice behavior in a touristy city: a factor analysis," Journal of Transport Geography, Online publishing, vol. 86, 2020.

[39] X. Li, W. Wang, C. Xu, Z. Li, and B. Wang, "Multi-objective optimization of urban bus network using cumulative prospect theory," Journal of Systems Science and Complexity, vol. 28, no. 3, pp. 661-678, 2015.

[40] D. H. Vu, K. M. Muttaqi, and A. P. Agalgaonkar, "A variance inflation factor and backward elimination based robust regression model for forecasting monthly electricity demand using climatic variables," Applied Energy, vol. 140, pp. 385394, 2015.

[41] A. Mouwen, "Drivers of customer satisfaction with public transport services," Transportation Research Part A: Policy and Practice, vol. 78, pp. 1-20, 2015. 